\title{
Longitudinal evaluation of corticospinal tract in patients with resected brainstem cavernous malformations using high-definition fiber tractography and diffusion connectometry analysis: preliminary experience
}

\author{
*Amir H. Faraji, MD, PhD, ${ }^{1}$ Kumar Abhinav, MD, ${ }^{1}$ Kevin Jarbo, BS, ${ }^{2}$ Fang-Cheng Yeh, MD, PhD, ${ }^{3}$ \\ Samuel S. Shin, MD, PhD, ${ }^{1}$ Sudhir Pathak, MS, ${ }^{2}$ Barry E. Hirsch, MD, ${ }^{4}$ Walter Schneider, PhD, ${ }^{2}$ \\ Juan C. Fernandez-Miranda, MD, ${ }^{1}$ and Robert M. Friedlander, MD, MA ${ }^{1}$ \\ Departments of ${ }^{1}$ Neurological Surgery and ${ }^{4}$ Otolaryngology, University of Pittsburgh Medical Center; ${ }^{2}$ Department of Psychology, \\ University of Pittsburgh; and ${ }^{3}$ Department of Biomedical Engineering, Carnegie Mellon University, Pittsburgh, Pennsylvania
}

\begin{abstract}
OBJECT Brainstem cavernous malformations (CMs) are challenging due to a higher symptomatic hemorrhage rate and potential morbidity associated with their resection. The authors aimed to preoperatively define the relationship of CMs to the perilesional corticospinal tracts (CSTs) by obtaining qualitative and quantitative data using high-definition fiber tractography. These data were examined postoperatively by using longitudinal scans and in relation to patients' symptomatology. The extent of involvement of the CST was further evaluated longitudinally using the automated "diffusion connectometry" analysis.
\end{abstract}

METHODS Fiber tractography was performed with DSI Studio using a quantitative anisotropy (QA)-based generalized deterministic tracking algorithm. Qualitatively, CST was classified as being "disrupted" and/or "displaced." Quantitative analysis involved obtaining mean QA values for the CST and its perilesional and nonperilesional segments. The contralateral CST was used for comparison. Diffusion connectometry analysis included comparison of patients' data with a template from 90 normal subjects.

RESULTS Three patients (mean age 22 years) with symptomatic pontomesencephalic hemorrhagic CMs and varying degrees of hemiparesis were identified. The mean follow-up period was 37.3 months. Qualitatively, CST was partially disrupted and displaced in all. Direction of the displacement was different in each case and progressively improved corresponding with the patient's neurological status. No patient experienced neurological decline related to the resection. The perilesional mean QA percentage decreases supported tract disruption and decreased further over the follow-up period (Case 1, 26\%-49\%; Case 2, 35\%-66\%; and Case 3,63\%-78\%). Diffusion connectometry demonstrated rostrocaudal involvement of the CST consistent with the quantitative data.

CONCLUSIONS Hemorrhagic brainstem CMs can disrupt and displace perilesional white matter tracts with the latter occurring in unpredictable directions. This requires the use of tractography to accurately define their orientation to optimize surgical entry point, minimize morbidity, and enhance neurological outcomes. Observed anisotropy decreases in the perilesional segments are consistent with neural injury following hemorrhagic insults. A model using these values in different CST segments can be used to longitudinally monitor its craniocaudal integrity. Diffusion connectometry is a complementary approach providing longitudinal information on the rostrocaudal involvement of the CST.

http://thejns.org/doi/abs/10.3171/2014.12.JNS142169

KEY WORDS diffusion MRI; brainstem cavernous malformations; corticospinal tract; fiber tractography; diffusion connectometry; quantitative anisotropy; vascular disorders

\footnotetext{
ABBREVIATIONS $\mathrm{ADL}=$ activities of daily living; $\mathrm{CC}=$ corpus callosum; $\mathrm{CM}=$ cavernous malformation; $\mathrm{CN}=$ cranial nerve; $\mathrm{CST}=\mathrm{corticospinal}$ tract; $\mathrm{DSI}=$ diffusion spectrum imaging; DTI = diffusion tensor imaging; FDR = false discovery rate; HDFT = high-definition fiber tractography; ICH = intracerebral hemorrhage; $\mathrm{QA}=$ quantitative anisotropy; $\mathrm{ROI}$ = region of interest; $\mathrm{SRS}=$ stereotactic radiosurgery.

SUBMITTED September 17, 2014. ACCEPTED December 8, 2014.

INCLUDE WHEN CITING Published online June 5, 2015; DOI: 10.3171/2014.12.JNS142169.

DISCLOSURE Funding was provided by the Pittsburgh Foundation (Copeland Fund), Fine Foundation, and the University of Pittsburgh Brain Institute.

* Drs. Faraji and Abhinav contributed equally to this work.
} 
$\mathrm{C}$ AVERNOUS malformations (CMs) are angiographically occult lesions with an estimated prevalence of $0.4 \%-0.8 \%$ in the general population. ${ }^{5,8}$ Most $\mathrm{CMs}$ occur in the supratentorial white matter, with the indications for their resection being controversial. ${ }^{38}$ Roughly $9 \%-35 \%$ of CMs are located in the brainstem ${ }^{35}$ and are believed to have higher symptomatic bleeding rates than those in the supratentorial compartment. The estimated bleeding and rebleeding rates for brainstem CMs range from $0.5 \%$ to $6 \%$ and $5 \%$ to $60 \%$ per patient-year, respectively. ${ }^{10,25,26,35}$ The management of brainstem CMs remains challenging due to a high degree of potential morbidity and mortality with and without treatment and specifically due to the eloquence of the perilesional anatomy. Resection is fraught with significant rates of permanent neurological deterioration, including for brainstem $\mathrm{CMs}$ reaching the pial surface. ${ }^{4,25}$ The increased surgical morbidity is partly due to uncertainty regarding the precise location of the affected white matter tracts. Preoperative identification of these tracts in their accurate spatial orientation may lead to a safer surgical corridor to reduce any potential morbidity.

Use of diffusion tensor imaging (DTI) in the resection of brainstem CMs with a higher annual average symptomatic hemorrhage rate of $2.7 \%-5 \%{ }^{4,20,32}$ has been reported. ${ }^{11,12,28,31,37,40}$ Its limitations include its inability to represent the crossing of multiple fibers or to determine with precision their cortical and subcortical terminations. ${ }^{7,24}$ DTI indices (e.g., fractional anisotropy [FA]) are susceptible to partial volume effects, leading to underestimation in areas with multiple crossing fibers. ${ }^{6,33}$ Despite these limitations, DTI has been an important adjunct in the resection of tumors ${ }^{23,51}$ and CMs. ${ }^{12,28,31,40}$

High-definition fiber tractography (HDFT), an advanced white matter imaging technique, addresses some of the limitations of DTI. HDFT is accurate in tracking fiber pathways through complex crossings to subcortical regions and targets. ${ }^{16}$ HDFT relies on diffusion spectrum imaging (DSI) for acquisition, ${ }^{44}$ generalized q-sampling imaging for fiber orientation estimation, and a generalized deterministic fiber tracking method. ${ }^{48}$ We have reported its use in studies on several white matter tracts ${ }^{18,19,41-43}$ and in a study concerning its utility for surgical planning for space-occupying lesions. ${ }^{16}$ Of direct relevance to this report, the utility of HDFT was recently reported in characterizing changes in the perilesional white matter and in guiding surgical planning in patients with supratentorial CMs using a combined qualitative and quantitative approach. ${ }^{1}$

In this report, we aimed to highlight the utility of HDFT in providing qualitative data with respect to accurately outlining the spatial orientation of the perilesional CST around the brainstem CMs. We demonstrate the usefulness of this information in guiding the resection of hemorrhagic pontomesencephalic CMs while minimizing injury to the motor tracts. ${ }^{16,17}$ We further aimed to characterize the perilesional changes in the CST quantitatively using quantitative anisotropy (QA) in line with our previously outlined methodology. ${ }^{1}$ This methodology focused on evaluating the integrity of the perilesional segment of an affected tract using QA. ${ }^{1}$ QA overcomes the limitations of FA in assessing the fiber integrity in areas with multiple crossing fibers since QA is less sensitive to partial volume effects, as demonstrated in our recent study. ${ }^{48}$

The resistance of QA to multiple crossing fibers also enabled us to aim to indirectly ascertain the craniocaudal integrity of the affected CST by obtaining quantitative information with respect to 3 rostrocaudal segments (perilesional and proximal and distal to the lesion). We longitudinally monitored the qualitative and quantitative tractography data, aiming to indirectly assess the craniocaudal integrity of an affected tract over time.

Finally, due to limitations associated with tractographybased studies and to validate our findings, we obtained longitudinal qualitative information regarding the involvement of the CST by adaptation of our so-called "diffusion connectometry" approach. ${ }^{2}$ This automated approach permitted comparison of our patients' data with a template created from the imaging data of healthy subjects to identify the affected tracts. Diffusion connectometry identifies tracts with decreased or increased connectivity in affected individuals (e.g., in patients with brainstem $\mathrm{CMs}$ ) compared with a normal population. The conventional tractography approach relies on choosing regions of interest (ROIs) according to the defined tracts of interest (e.g., CST) before comparing the difference, making it vulnerable to reproducibility issues. Diffusion connectometry overcomes these problems since it is an automated technique that does not involve manual selection of the ROIs and finds the local differences in the diffusion distribution without initiating fiber tracking, making it a more robust approach for detecting any real difference in the connectivity. Further details regarding the basic methodology and its adaptation to analyze the longitudinal data can be referred to in our studies on stroke and amyotrophic lateral sclerosis patients. ${ }^{2,46}$ It is noteworthy that this is the first report using connectometry analysis in the evaluation of a lesional pathology.

\section{Methods}

\section{Patient Descriptions}

All 3 patients were screened to rule out any contraindications to the longitudinal MRI scans. Consent was obtained in accordance with the University of Pittsburgh's Institutional Review Board. Analysis of the data was performed with the reviewer blinded to the patients' clinical details. The senior author (R.M.F.) performed the resection of the $\mathrm{CM}$ in all cases.

\section{Image Acquisition and Reconstruction}

DSI data were acquired using a 3-T Tim Trio System (Siemens) with a 32-channel coil. A head stabilizer prevented head motion. A 43-minute, 257-direction DSI scan with a twice-refocused spin-echo planar imaging sequence and multiple b values (TR $9916 \mathrm{msec}$, TE $157 \mathrm{msec}$, voxel size $2.4 \times 2.4 \times 2.4 \mathrm{~mm}^{3}$, field of view $231 \times 231 \mathrm{~mm}^{2}$, maximum b-value $7000 \mathrm{sec} / \mathrm{mm}^{2}$ ) was performed. For anatomical comparisons, we included high-resolution anatomical imaging using a 9-minute T1-weighted axial magnetization-prepared rapid-acquisition gradient-echo sequence (TR $2110 \mathrm{msec}$, TE $2.63 \mathrm{msec}$, flip angle $8^{\circ}$, 
number of slices 176 , field of view $256 \times 256 \mathrm{~mm}^{2}$, voxel size $0.5 \times 0.5 \times 1.0 \mathrm{~mm}^{3}$ ). DSI data were reconstructed with a generalized q-sampling imaging approach. The orientation distribution functions were reconstructed to 642 discrete sampling directions for each pixel and with a diffusion distance scaling parameter of 1.2.

\section{Tractography-Based Approach \\ Fiber Tracking}

DSI Studio (dsi-studio.labsolver.org) was used to visualize the left and right CST from the DSI data sets using a generalized deterministic tractography method. ${ }^{48} \mathrm{~A}$ whole brain seeding approach using multiple, single-slice ROI and region of avoidance masks was implemented. For CST reconstruction, 2 ROIs were drawn in the axial plane to cover the white matter of the medulla and the posterior limb of the internal capsule just superior to the midbrain in each hemisphere. Each mask was drawn on a generalized FA map in tissue unaffected by the lesion or the resection procedure. These ROIs were kept constant across all subjects and longitudinal scans. A region of avoidance was drawn over the corpus callosum (CC) at the sagittal midline to eliminate the false continuation of CST fibers to the contralateral hemisphere. DSI Studio provides a default QA threshold to enable tractography. This default threshold can be adjusted to optimize the white matter coverage. This threshold was then kept constant across the longitudinal scans for the individual subject for depicting the CST. Fiber tracking was initiated from the whole brain seed using all orientations present within a voxel until 20,000 streamlines were detected. To maximize the detection of CST, the following parameters were kept constant across all subjects and the longitudinal scans: maximum turning angle of $90^{\circ}$, step size of $0.5 \mathrm{~mm}$, smoothing 0.8 , and a length constraint of $100-200 \mathrm{~mm}$. All fibers terminating outside the precentral gyrus were deleted. In our experience, using a high turning angle allows a better depiction of the CST fibers originating from the lateral aspect of the precentral gyrus.

\section{Qualitative Analysis}

We described the CST as being disrupted and/or displaced using the contralateral homologous CST for comparison. " Disruption" may be partial, e.g., due to "breakage" or thinning of fibers as seen in a cross-section or due to discontinuity in a limited segment of the tract, or complete if the tract was not seen through most or all of its course. Definition of "displacement" was strictly limited to a change of location or direction in the affected tract due to the lesion-related mass effect. ${ }^{23}$ The qualitative changes were monitored longitudinally.

\section{Quantitative Analysis}

Quantitative analysis followed qualitative analysis and was also carried out in a longitudinal fashion. Mean QA values were obtained for the whole CST on both sides (Table 1). We further used mean QA values for the perilesional segments (Table 2 and Supplemental Table) to check for consistency with the observed qualitative change, as outlined in our previous study (see Supplemental Material, available online) on supratentorial CMs. ${ }^{1}$

\section{Diffusion Connectometry Approach}

In this study, we applied diffusion MRI connectometry analysis $^{46}$ to evaluate the affected motoric tracts in relation to those in healthy subjects. The diffusion data of each subject are reconstructed in a common stereotactic space using the q-space diffeomorphic reconstruction..$^{47}$ The data of the brainstem in CM patients were compared with that of 90 normal subjects (using the NTU90 Template in MNI Space provided by DSI Studio; mean age 33 years). ${ }^{47} \mathrm{~A}$ percentile threshold of 5\% was used to identify fiber orientations with substantial decrease in diffusion. Deterministic fiber tracking was used to connect these orientations. The length threshold allows differentiation of meaningful findings from random variations. ${ }^{2,46}$ Normal subjects may have false-positive affected tracts due to the noise in the diffusion-weighted images, resulting in fragmentation of the tracts. In affected patients (e.g., those with brainstem $\mathrm{CMs}$ ) the true connectivity difference will continue along the fiber pathways, forming longer affected tracts. Using length as an index (length threshold), the difference between the random effect and true connectivity difference is statistically tested. The determination of this threshold is a trade-off between sensitivity and specificity with findings becoming more specific with a higher threshold. Based on our previous studies ${ }^{2,46}$ and the current aim to detect changes in the CST, a length threshold of $40 \mathrm{~mm}$ was used, resulting in a false discovery rate (FDR) of 0.12 (Fig. 1). This means that approximately $12 \%$ of the affected tracts may be identified as being falsely affected. To track the longitudinal data, we used the same length threshold and, therefore, the FDR to examine the subsequent scan. ${ }^{2} \mathrm{We}$ hypothesized that any demonstrated change with respect to the visualized affected tracts was real, and therefore an indirect marker of deterioration or improvement in those tracts in patients with brainstem CMs.

These qualitative changes were represented using the MNI T1-weighted template in the DSI Studio. The affected tracts, as demonstrated by the connectometry, only reveal the affected segments of the entire tract, which were confirmed to be CST based on their anatomical location and using the "tracts to ROI" function in DSI Studio. Using this function, the end points of the affected segments were projected onto the cortical gyri to confirm their origin from the precentral gyrus. We also examined the segments of the affected CST and their change, as revealed by the connectometry in relation to the quantitative data for the specific CST segments from the tractography-based analysis.

\section{Results}

\section{Case Details and Tractography-Based Qualitative Data Case 1}

A 24-year-old woman experienced a hemorrhage from a left pontomesencephalic CM and was admitted to another institution with a dense right upper- and lower-extremity hemiparesis, a right gaze preference, and speech apraxia. Two weeks later, the patient's condition clinically deteriorated and she was emergently transferred to our institution. On examination, the patient was fully oriented with a moderate speech apraxia, right facial droop, diplopia on left lateral gaze, and right-sided tongue deviation. 
TABLE 1. Overall mean QA values for the CST over longitudinal scans

\begin{tabular}{cclccc}
\hline $\begin{array}{c}\text { Case No. } \\
\text { (affected side) }\end{array}$ & Scan No. (stage) & Time Period & Mean QA \pm SD on the Rt & Mean QA \pm SD on the Lt & \% Decrease $(Q A)^{*}$ \\
\hline 1 (It) & 1 (preop) & Immediately & $0.16 \pm 0.08$ & $0.14 \pm 0.08$ & $12.83 \%$ \\
\hline & 2 (postop) & 3 wks & $0.17 \pm 0.08$ & $0.12 \pm 0.06$ & $26.57 \%$ \\
\hline 2 (It) & 3 (postop) & 6 mos & $0.15 \pm 0.06$ & $0.10 \pm 0.06$ & $28.47 \%$ \\
\hline & 1 (preop) & Immediately & $0.19 \pm 0.08$ & $0.14 \pm 0.08$ & $27.24 \%$ \\
\hline 3 (rt) & 2 (postop) & Immediately & $0.21 \pm 0.09$ & $0.14 \pm 0.08$ & $35.29 \%$ \\
\hline & 1 (preop) & 1 year & $0.07 \pm 0.04$ & $0.10 \pm 0.05$ & $35.87 \%$ \\
\hline & 3 (preop) & Immediately & $0.05 \pm 0.03$ & $0.10 \pm 0.03$ & $52.46 \%$ \\
\hline & 4 (postop) & Immediately & $0.05 \pm 0.03$ & $0.10 \pm 0.05$ & $52.32 \%$ \\
\hline
\end{tabular}

${ }^{*}$ Calculated for the affected side in relation to the unaffected side.

Her strength, as assessed by the British Medical Research Council scale, was Grade 5/5 in both the left upper and lower limbs. In the right upper limb, the strength was recorded as Grade 4/5 in the proximal right upper extremity and Grade 0/5 with a trace of finger flexion in the distal right upper extremity. In the right lower limb, her proximal strength was Grade 4/5, while distally it was Grade 1/5. Examination also revealed dysmetria (finger-to-nose testing) and hyperreflexia. There were no sensory deficits.

MRI demonstrated a hemorrhagic CM (Fig. 2) in the anterolateral pons with extension into the middle cerebral peduncle and midbrain asymmetrically with left-sided predominance. Preoperative HDFT (Figs. 2A and 6A) indicated partial disruption and posterior displacement of the left CST fibers. A left anterior subtemporal transtentorial approach after an extradural anterior petrosectomy was performed. ${ }^{27}$ The anterolateral surface of the mid- brain revealed a subtle yellow stain, which was chosen as the entry point to avoid the perilesional CST fibers with their known posterior displacement. A minimal opening was made into the midbrain to evacuate the hematoma and resect the $\mathrm{CM}$.

The patient's immediate postoperative examination revealed improved cranial nerve (CN) IV and VI palsies with resolving diplopia, facial droop, and dysarthria. Her strength was Grade 4/5 in the proximal right upper extremity and improved to Grade 1/5 with a trace of finger flexion in the distal right upper extremity. In the right lower extremity, her proximal strength was Grade $4 / 5$, while distally it improved to Grade 2/5. Sensory examination findings were normal. Postoperative MRI was correlated with a 3-week postoperative HDFT study to evaluate the impact of resection on the CST (Fig. 2B), which demonstrated reduced displacement. At the 6-month follow-up,

TABLE 2. Qualitative change and percentage decreases in the mean QA values for the CST segments over longitudinal scans in relation to the motor findings*

\begin{tabular}{|c|c|c|c|c|c|c|c|}
\hline $\begin{array}{c}\text { Case No. } \\
\text { (affected side) }\end{array}$ & $\begin{array}{l}\text { Scan } \\
\text { No. }\end{array}$ & Stage & $\begin{array}{l}\text { Segment } 1 \\
\text { (proximal) }\end{array}$ & $\begin{array}{c}\text { Segment } 2 \\
\text { (perilesional) }\end{array}$ & $\begin{array}{l}\text { Segment } 3 \\
\text { (distal) }\end{array}$ & Qualitative Change & $\begin{array}{l}\text { Limb Motor Findings } \\
\text { (MRC grade) }\end{array}$ \\
\hline \multirow[t]{3}{*}{$1(\mathrm{It})$} & 1 & Immediately preop & $8.44 \%$ & $26.38 \%$ & $32.89 \%$ & $\begin{array}{l}\text { Partial disruption \& displace- } \\
\text { ment (posterior) }\end{array}$ & $\begin{array}{l}\mathrm{RUL}(4 / 5 \text { proximal \& } 0 / 5 \text { distal) \& } \\
\mathrm{RLL}(4 / 5 \text { proximal \& } 1 / 5 \text { distal) }\end{array}$ \\
\hline & 2 & 3 wks postop & $26.84 \%$ & $28.12 \%$ & $20.16 \%$ & $\begin{array}{l}\text { Partial disruption \& reduced } \\
\text { displacement (posterior) }\end{array}$ & $\begin{array}{l}\text { RUL (4/5 proximal \& } 1 / 5 \text { distal) \& } \\
\text { RLL (4/5 proximal \& } 2 / 5 \text { distal) }\end{array}$ \\
\hline & 3 & 6 mos postop & $24.17 \%$ & $48.70 \%$ & $45.59 \%$ & Partial disruption only & $\begin{array}{l}\mathrm{RUL}(4+/ 5) \& \mathrm{RLL}(4+/ 5 \text { proximal } \\
\quad \& 3 / 5 \text { distal) }\end{array}$ \\
\hline \multirow[t]{2}{*}{$2(\mathrm{lt})$} & 1 & Immediately preop & $22.68 \%$ & $34.70 \%$ & $31.62 \%$ & $\begin{array}{l}\text { Partial disruption \& displace- } \\
\text { ment (AM) }\end{array}$ & RUL \& RLL (4/5) \\
\hline & 2 & Immediately postop & $22.42 \%$ & $65.72 \%$ & $48.38 \%$ & $\begin{array}{l}\text { Partial disruption w/ residual } \\
\text { displacement }\end{array}$ & RUL \& RLL (4+/5) \\
\hline \multirow[t]{4}{*}{$3(\mathrm{rt})$} & 1 & 1 yr preop & $14.68 \%$ & $63.39 \%$ & $42.20 \%$ & $\begin{array}{l}\text { Partial disruption \& displace- } \\
\text { ment (lat) }\end{array}$ & None \\
\hline & 3 & Immediately preop & $45.06 \%$ & $77.70 \%$ & $42.77 \%$ & $\begin{array}{l}\text { Partial disruption \& displace- } \\
\text { ment (PL) }\end{array}$ & $\begin{array}{l}\text { LUL }(4 / 5 \text { proximal } \& 0 / 5 \text { distal }) \& \\
\quad \text { LLL }(4+/ 5)\end{array}$ \\
\hline & 4 & Immediately postop & $44.66 \%$ & $76.62 \%$ & $27.94 \%$ & $\begin{array}{l}\text { Partial disruption \& improving } \\
\text { displacement }\end{array}$ & LUL (1/5) \& LLL (4/5) \\
\hline & 5 & 4 mos postop & $46.06 \%$ & $78.52 \%$ & $66.87 \%$ & Partial disruption only & $\begin{array}{l}\text { LUL (4/5 proximal \& 2-3/5 distal) } \\
\text { \& LLL (4/5) }\end{array}$ \\
\hline
\end{tabular}

$\mathrm{AM}=$ anteromedial; $\mathrm{LLL}$ = left lower limb; $\mathrm{LUL}$ = left upper limb; $\mathrm{MRC}=$ Medical Research Council; $\mathrm{PL}$ = posterolateral; $\mathrm{RLL}$ = right lower limb; RUL = right upper limb.

* Percentage decreases are calculated for the affected segments in relation to the unaffected segments. 


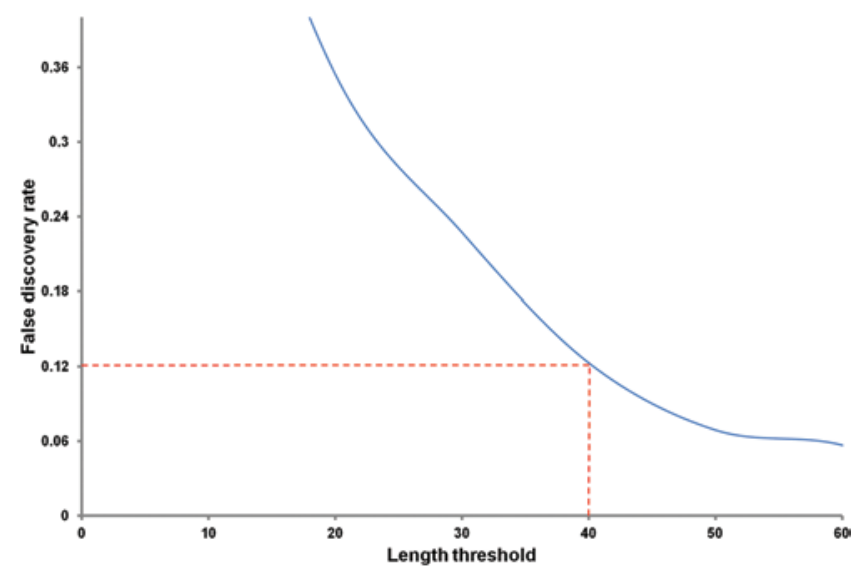

FIG. 1. Length threshold in relation to the FDR. A length threshold of 40 $\mathrm{mm}$ was chosen, corresponding to an FDR of 0.12 (denoted by the dotted line). Figure is available in color online only.

there was improvement in the patient's neurological status corresponding with the resolution of displacement of the CST on the HDFT (Fig. 2C). A neurological examination revealed a slight facial droop, Grade 4+/5 strength in the right upper limb and the proximal right lower limb, and Grade 0/5 strength in dorsiflexion and Grade 3/5 in plantar flexion in the distal right lower limb. The patient was independent with her activities of daily living (ADL). Additionally, she was a skilled right-handed artist prior to the hemorrhage and had regained the ability to draw at the last follow-up. Four years and 2 months after resection of the $\mathrm{CM}$, there is no evidence of recurrence or rehemorrhage.

\section{Case 2}

A 20-year-old man presented with a 1-week history of left-sided headache as well as right-sided weakness and paresthesias. On examination, the patient was fully oriented. Left CN IV, VI, and VII palsies were observed, with decreased sensation (to light touch) in the right $\mathrm{CN}$ V2 distribution. There were no sensory or motor deficits in the left upper and lower limbs. In the right upper and lower limbs, examination revealed increased tone, Grade $4 / 5$ strength, and decreased sensation to light touch. The patient also had mild urinary retention. MRI indicated the presence of a left-sided posterolateral pontomesencephalic hemorrhagic CM. HDFT indicated anteromedial displacement (across the anterior surface of the pons) and partial disruption of the left CST (Figs. 3A and 6B). An apical petrosectomy and a left-sided subtemporal transtentorial approach were performed to resect the CM. Using the image guidance and the information from the tractography, the surgical entry point was made along the posterolateral aspect of the pons to resect the hemorrhagic CM. The patient's immediate postoperative examination revealed only a partial left CN VI palsy with resolving diplopia. His strength had improved to Grade $4+/ 5$ in the right upper and right lower extremities. Sensation to all modalities was normal. An immediate postoperative HDFT study demonstrated improvement in the configuration of the left CST with some residual displacement (Figs. 3B and 6B). Over the next 3 months, the patient's strength remained stable in the right upper and lower limbs at Grade $4+/ 5$ along with complete resolution of his double vision. One year after surgery, he had full strength and was independent with his ADL. Three years and 2 months following resection of the $\mathrm{CM}$, there is no evidence of recurrence or rehemorrhage.

\section{Case 3}

A 22-year-old woman presented with headache and transient left hemibody paresthesia and dysesthesias. A CT scan of the brain demonstrated a $2 \times 2-\mathrm{cm}$ right thalamic hematoma with mass effect with a negative angiographic workup. One week later, the patient experienced acuteonset left leg weakness, which resolved over several hours. MRI demonstrated a resolving hematoma without any conclusive etiology. Due to the potential surgical morbidity and symptomatic resolution, the patient was managed conservatively with planned radiological surveillance.

MRI at the 3-month follow-up demonstrated a $1.7-\mathrm{cm}$ thalamic hematoma extending into the cerebral peduncle with evidence of hemosiderin deposition consistent with a CM. HDFT revealed a laterally displaced and partially disrupted CST (Figs. 4A, 5A, and 6C). The patient was treated with stereotactic radiosurgery (SRS) in light of the lesion's location and her stable neurological condition.

A month after SRS, the patient suffered a further mild symptomatic thalamic hemorrhage. Three months after SRS, the patient presented again with increasing headache, left hemibody paresthesia, and distal left lower-extremity weakness. CT imaging demonstrated a new 5-ml hemorrhage of the $\mathrm{CM}$ with perilesional edema extending into the right posterior limb of the internal capsule. A
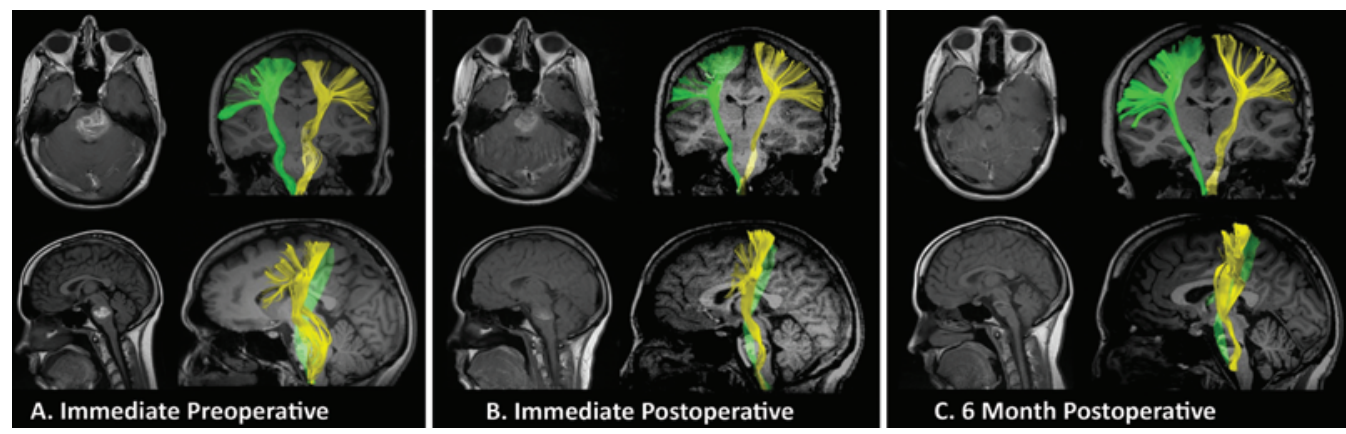

FIG. 2. Case 1. Longitudinal HDFT reconstructions of the CST in a patient with a left-sided pontomesencephalic CM. Immediate preoperative (A), immediate postoperative (B), and 6-month postoperative (C) scans demonstrating improvement in the displacement of the CST on the lesional side (yellow) in relation to the CST on the unaffected side (green) during the postoperative and follow-up periods. Figure is available in color online only. 

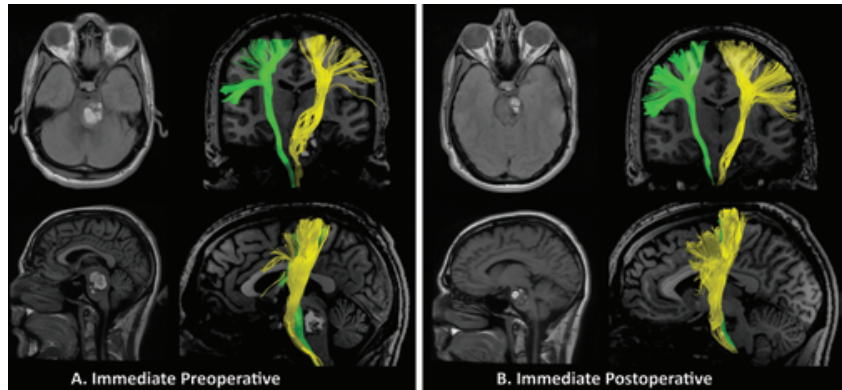

FIG. 3. Case 2. Pre- and postoperative HDFT reconstructions of the CST in a patient with a left-sided pontomesencephalic CM. Immediate preoperative (A) and postoperative (B) tractography studies of the CST showing an improvement in the configuration (displacement) of the perilesional CST (yellow) in relation to the contralateral CST (green) after resection of the $\mathrm{CM}$. Figure is available in color online only.

Solu-Medrol taper was initiated, and the patient's weakness resolved, although with persistent mild hemibody paresthesias. A full year since her initial presentation, the patient presented again with increasing headaches and left upper- and lower-extremity weakness. On examination, the patient was fully oriented. There was evidence of left CN III and VII palsies, Grade 4/5 strength in both the left upper and lower limbs, and decreased sensation to light touch over the left hemibody. CT scanning demonstrated a persistent right-sided thalamic and mesencephalic $\mathrm{CM}$ with increased perilesional edema. A corresponding HDFT (Figs. 4B and 5B) revealed further displacement and deformation of the CST with fibers visualized across the lateral aspect of the hemorrhagic CM. Given the patient's multiple hemorrhagic episodes and deteriorating findings on neurological examination, surgical intervention was strongly recommended.

One week later while considering surgery, the patient's left upper-limb strength acutely deteriorated to Grade 4/5 proximally and Grade 0/5 distally. Her left lower-limb strength remained at Grade 4+/5. A further HDFT (Figs. $4 \mathrm{C}$ and 5C) and MRI revealed an increase in the size of the CM-associated hemorrhage with continued displacement of the CST along the posterolateral aspect of the lesion. The patient underwent an image-guided right subtemporal approach to resect the mesencephalic CM. Hemosiderinstained tissue and hematoma were readily encountered at the anterolateral portion of the mesencephalon. Using the information from the HDFT, an anterior pial opening was made to prevent injury to the posterolaterally displaced CST. The patient's immediate postoperative examination revealed a partial left CN III palsy and slight left CN VII palsy, Grade 1/5 strength in the left upper and Grade 4/5 in the left lower limbs, and decreased sensation to all modalities in the left hemibody. Immediate postoperative MRI and HDFT (Fig. 4D) demonstrated complete resection of the $\mathrm{CM}$ and improvement in the displacement of the right CST, respectively. The patient had continuing neurological improvement, such that she was ambulating with a cane at 1 month postoperatively. At the 4-month postoperative follow-up, examination revealed complete resolution of the CN deficits and Grade 4/5 strength in the left lower limb and proximal upper limb. Distally in the left upper limb the strength in her finger extensors was Grade 2/5 and that in her finger flexors was Grade 3/5. The patient was independent with her ADL and ambulating without an aid. Two years following surgery there is no evidence of rehemorrhage or recurrence of the CM. Another HDFT scan (Fig. 4E) revealed complete resolution of the displacement of the partially disrupted CST, corresponding with her improved neurological status.

\section{Relevant Scans for the Tractography-Based Quantitative Data and Diffusion Connectometry Approach}

The quantitative data and the diffusion connectometry approach-based results are presented with respect to the following HDFT scans for 3 patients: Case 1 (Scan 1, immediately preoperative; Scan 2, 3 weeks after surgery; and Scan 3, 6 months after surgery), Case 2 (Scan 1, immediately preoperative; and Scan 2, immediately postoperative phase), and Case 3 (Scan 1, 1 year prior to surgery; Scan 3 , immediately preoperative phase; Scan 4 , immediately postoperative phase; and Scan 5, 4 months following surgery).

\section{Tractography-Based Quantitative Data Mean QA Values for the Whole CST}

Overall mean QA values (Table 1) were generally noted to be lower for the CST on the affected side for all patients. At the initial HDFT scan, the percentage difference in the QA values between the 2 sides was found to be outside the normal difference range obtained from the template data
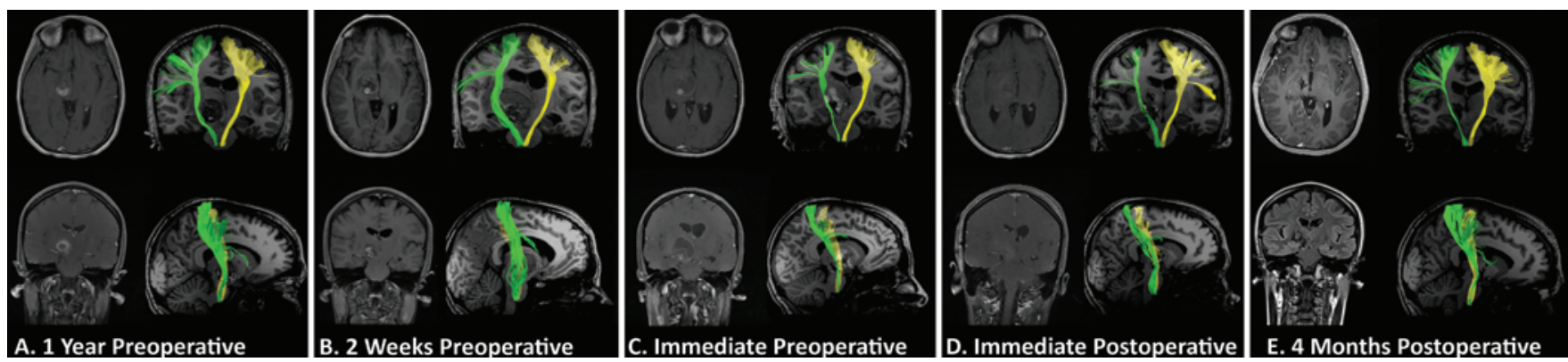

FIG. 4. Case 3. Longitudinal HDFT reconstructions of the CST in a patient with a right-sided thalamomesencephalic CM. A-C: Preoperative HDFT reconstructions of the CST demonstrating a displaced and partially disrupted perilesional CST (green) in relation to the $\mathrm{CM}$. There is worsening of the displacement between the first (A) and the second (B) HDFT scans. D and E: In the immediate postoperative phase (D) and the follow-up period $(E)$, there is an improvement in the configuration of the previously displaced CST in relation to the unaffected CST (yellow). Figure is available in color online only. 

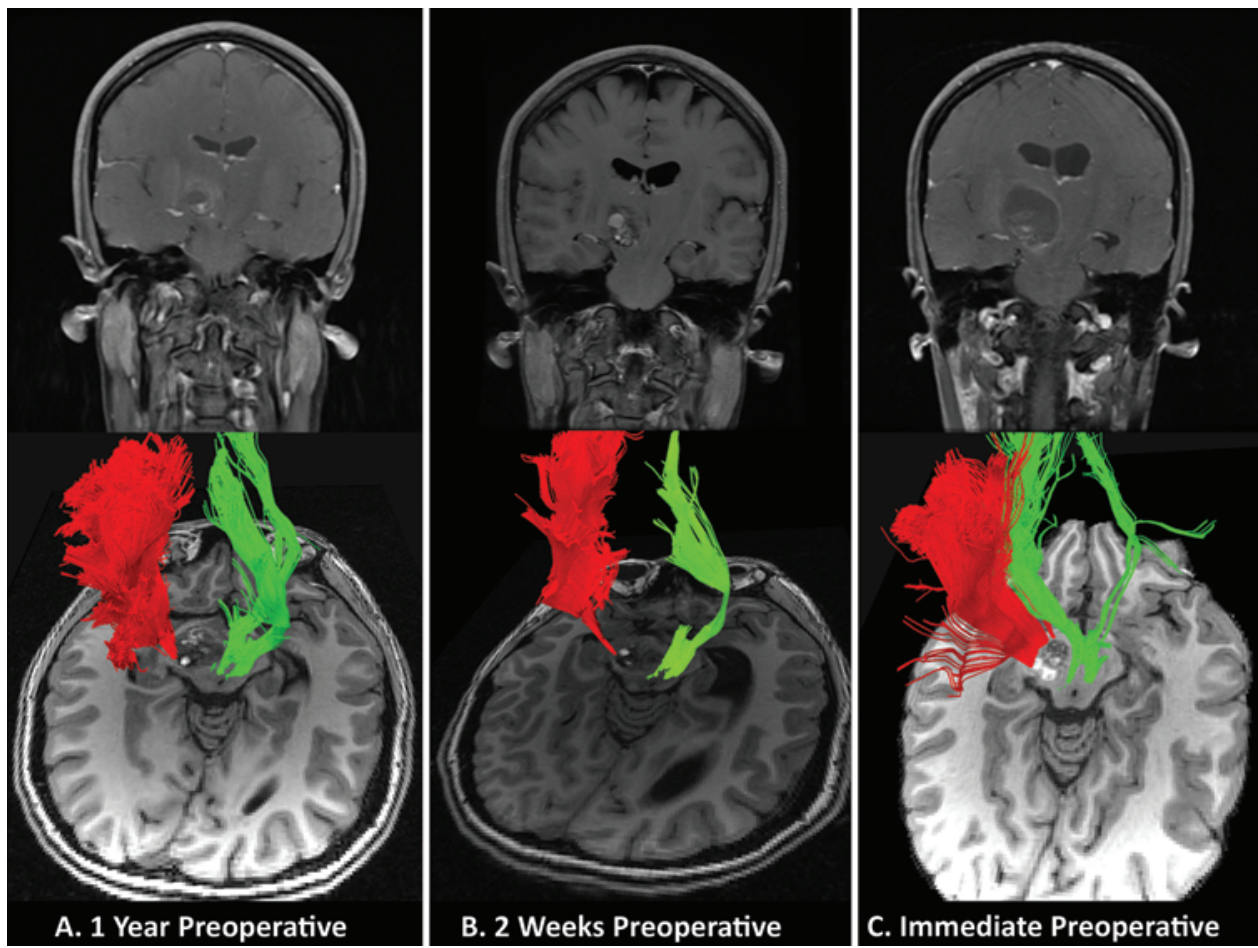

FIG. 5. Case 3. Longitudinal preoperative HDFT reconstructions of the perilesional tracts in a patient with a right-sided thalamomesencephalic CM (represented using axial MR slices). Preoperative HDFT reconstructions of the CSTs demonstrate a laterally and partially disrupted perilesional CST (red) in relation to the CM. There is progressive worsening of the fiber configuration, with the immediate preoperative scan (C) demonstrating posterolateral displacement of the CST. This led to selection of an anterior trajectory to resect the CM and to minimize any injury to the CST. In relation to the $\mathrm{CM}$, medially located superior cerebellar peduncle and medial lemniscus (green) fibers are also demonstrated. Figure is available in color online only.

for all patients. Specifically for Case 1, there was a further decrease in the values postoperatively, followed by stabilization at the 6-month follow-up. For Case 2, the differences in the QA values across the 2 scans were relatively stable. In Case 3, the difference was higher at baseline followed by deterioration in the immediately preoperative phase (Scan 3). After surgery and over the follow-up period, the difference in the values between the 2 sides remained relatively stable in Case 3 .

\section{Percentage Differences in Mean QA Values for the CST Segments}

In all patients (Table 2 and Supplemental Table), the percentage differences (decrease) in the mean QA values for the perilesional segments in relation to the unaffected side were higher or outside the range from the control group analysis. This was true across the longitudinal data and was also largely applicable to the nonperilesional segments. Further deterioration in these values was noted for the tract segments over time, particularly so for the perilesional and the distal segments. Of note, there was no improvement in the observed decrease for the perilesional segment after resection of the lesion and removal of the blood products.

\section{Diffusion Connectometry Approach-Based Qualitative Results}

In all patients, the affected CST as revealed by the automated connectometry analysis corresponded appro- priately to the lesional side. For the patient in Case 1, the connectometry analysis at the first scan (preoperative) revealed the affected segment of the CST (Fig. 7A) as being predominantly caudal, approximating predominantly to the level of the lesion and to a small distal segment beyond the lesion. At the second scan, 3 weeks after surgery (Fig. 7A) the CST was affected more rostrally with the most caudal segment now not seen as being affected. At the third scan, 6 months after surgery, there was extension in the involvement of the CST in the craniocaudal direction (Fig. 7A). With the patient in Case 2, the connectometry analysis of both the pre- and postoperative scans demonstrated a similar rostrocaudal extent of involvement of the CST (Fig. 7B). The affected overall segment of the CST as demonstrated approximated to the level of the lesion and segments both proximal and distal to the lesion. In the patient in Case 3 with a thalamomesencephalic lesion, both the preoperative (Scans 1 and 3, Fig. 7C) and the first postoperative scan (Scan 4, Fig. 7D) demonstrated extensive rostrocaudal involvement of the CST with changes also being observed in the body of the $\mathrm{CC}$. These changes were also present at the last scan 4 months after surgery (Fig. 7D) with the rostral CST on the contralateral side also seen as being affected.

\section{Discussion}

\section{Brainstem CMs in General}

Brainstem CMs have a high degree of morbidity and 


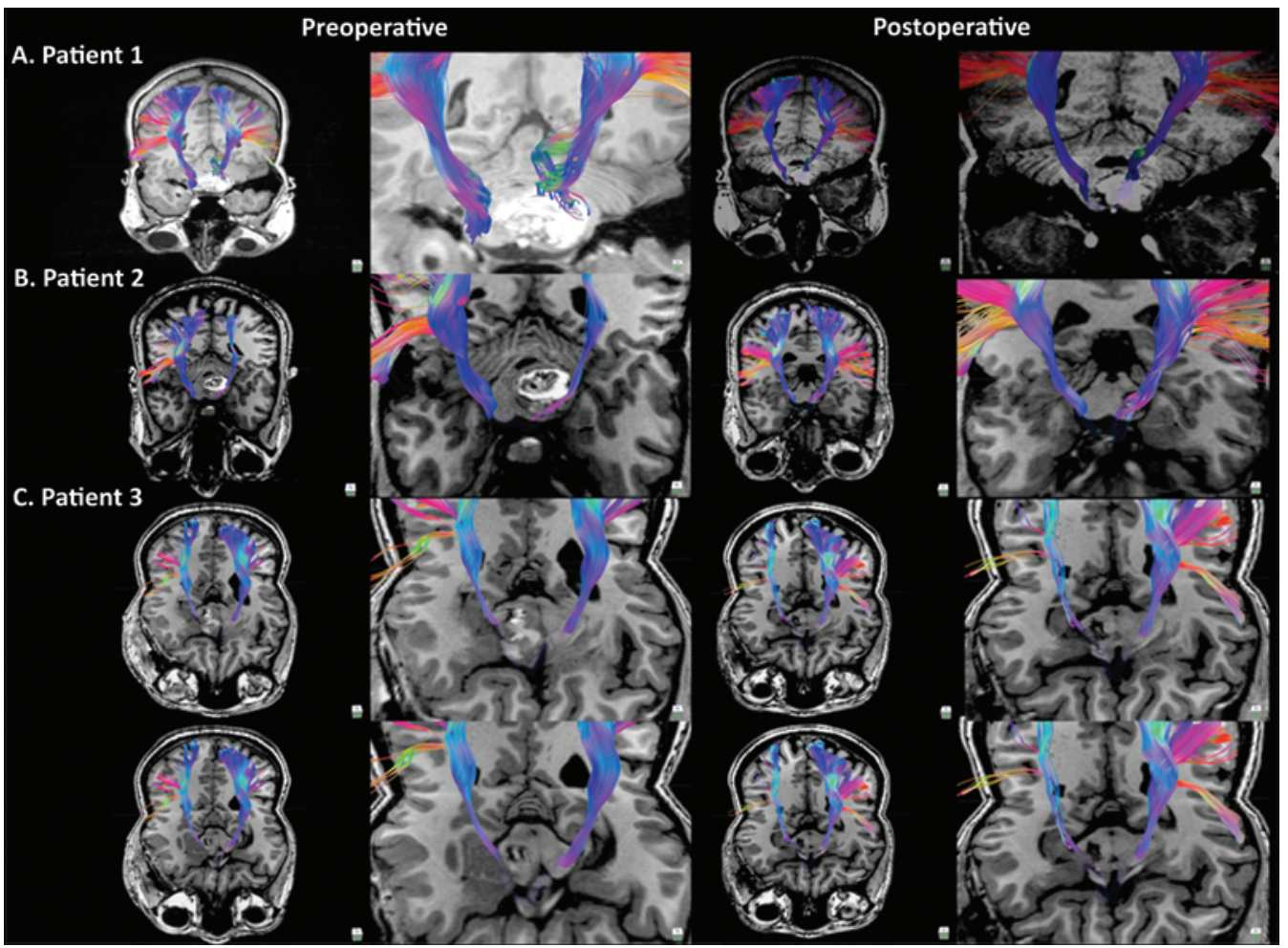

FIG. 6. Demonstration of deformation of the pre- and postoperative CST in relation to the CM (magnified views) using the HDFT. The direction of displacement of CST was different for the 3 cases. Case 1 (A) had a posteriorly displaced left CST, Case 2 (B) had an anteromedially displaced left CST, and Case 3 (C) had a laterally displaced right CST in relation to the lesion. Figure is available in color online only.

mortality associated with both their natural history and treatment. Unlike brainstem CMs, supratentorial CMs without a history of prior bleeding have only a $0.6 \%$ per year risk of hemorrhage. ${ }^{32}$ In a recent study evaluating the hemorrhage risks of untreated brainstem CMs, the annual hemorrhage rates in patients initially presenting with hemorrhage and focal neurological deficits was $15.9 \% .^{26}$

Patients with brainstem CMs can be managed conservatively or treated with radiosurgery ${ }^{32}$ and/or resection. Although the risk of postoperative rehemorrhage following surgery was reduced to $2.0 \%$ per year in one series, high rates of new postsurgical deficits are routinely reported. ${ }^{21,25,35}$ Despite advances in the microsurgical techniques, in a large single-center series of 260 adult patients with surgically treated brainstem CMs, $53 \%$ of the patients developed new or worsening neurological symptoms postoperatively; these neurological deficits were permanent in $36 \%{ }^{4}$ This is not surprising in light of the eloquent perilesional white matter.

In cases presenting with acutely symptomatic hemorrhagic brainstem $\mathrm{CM}$, resection ${ }^{28}$ may be the most effective treatment strategy to address the mass effect and reduce the risk of further symptomatic hemorrhage and associated worsening of the neurological deficit.

\section{Tractography-Based Qualitative Data and Implications}

DTI studies ${ }^{11,12,28,31,37,40}$ in general have qualitatively demonstrated the orientation of the eloquent white matter tracts (deviated and partially interrupted) around the brainstem CMs for planning the surgical trajectory. A re- cent study ${ }^{40}$ using DTI-based tractography retrospectively analyzed the pre- and postoperative visualization of the CST and sensory tracts in 23 cases. The improvement in visualization was associated with the patients' neurological status. Consistent with other reports, ${ }^{1,11} \mathrm{CMs}$ had caused a combination of displacement, thinning, or interruption of the fiber tracts.

In our cases, the qualitative data (Figs. 2-6 and Table 2) precisely outlined the spatial orientation of the affected CST. The surgical trajectory was chosen based on the direction of the displacement of the fibers, which occurred in a different direction (Fig. 6) for each patient (Case 1, posterior; Case 2, anterior; and Case 3, initially lateral and then posterolateral after several hemorrhagic events). Consistent with other studies and our previous findings from the supratentorial CM study, we found a combination of displacement and partial disruption occurring in the CST adjacent to the brainstem CMs., ${ }^{111}$ The presence and improvement of motor deficits correlated with the observed displacement in the CST and its improvement over time, respectively (Table 2). Accurate depiction of the perilesional CST including in the edematous zone using HDFT is linked in part to the QA being less sensitive to partial volume effects. 3.48

The surgical planning in our studies ${ }^{1,15,16}$ for mass lesions has been based on the principle of avoidance of damage to the displaced tracts as a result of the initial surgical trajectory or eventual lesionectomy. This is related to the hypothesis that displaced tracts, even though functionally compromised, retain the ability to recover after removal 

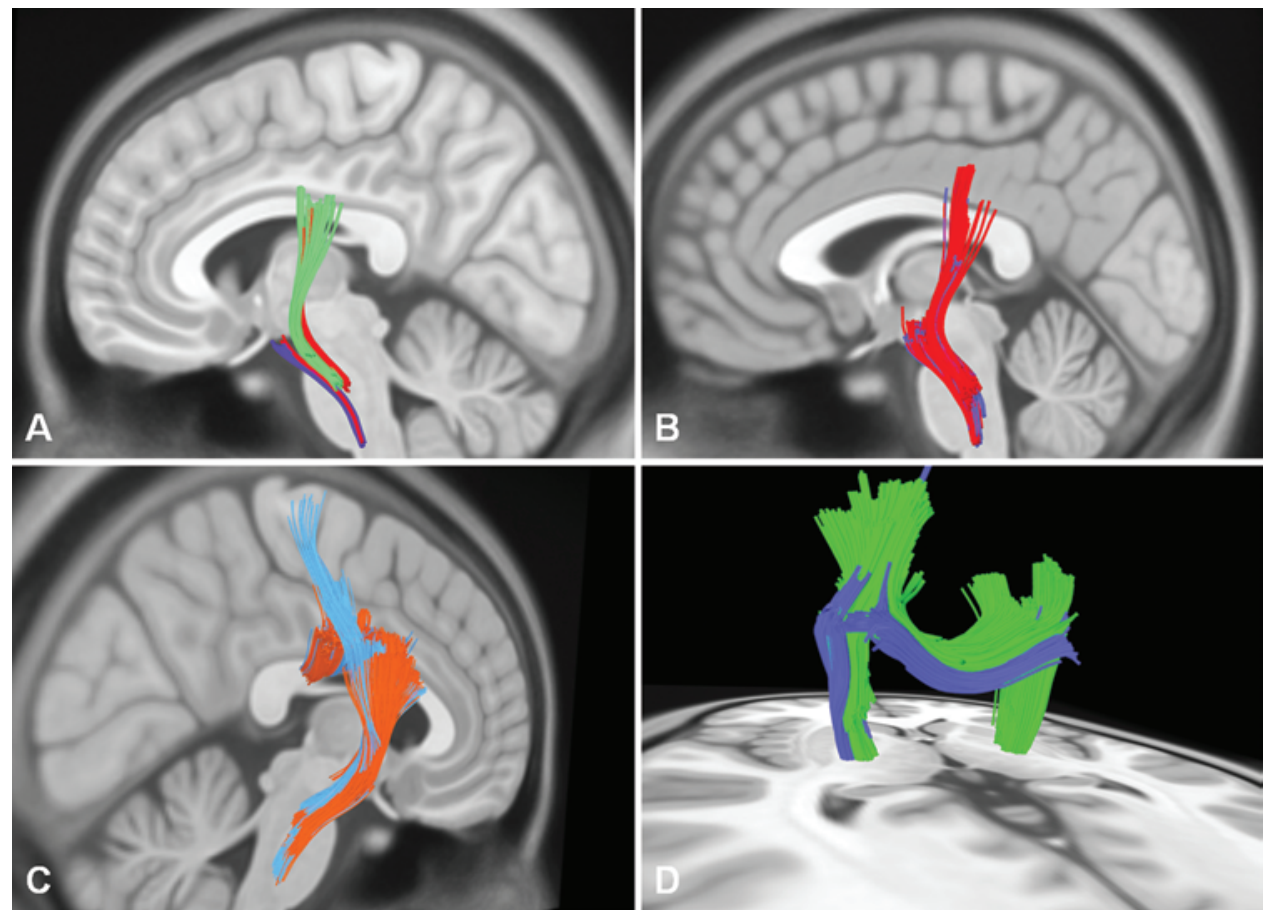

FIG. 7. Diffusion connectometry analysis approach in Cases 1 (A), 2 (B), and 3 (C and D). A: For Case 1 with a left-sided pontomesencephalic CM, the connectometry analysis at the first scan (preoperative, blue) revealed the affected segment of the CST as being predominantly caudal, approximating to the level of the lesion with the second scan ( 3 weeks after surgery, green) indicating a more rostral involvement. At the third scan ( 6 months after surgery, red), there was an increase in the extent of the involvement of CST in the craniocaudal direction. B: For Case 2 with a left-sided pontomesencephalic CM, the connectometry analysis of both the immediately pre- (blue) and postoperative (red) scans demonstrated similar rostrocaudal involvement of the CST. C and D: In Case 3 with a right-sided thalamomesencephalic lesion, both the preoperative (C, blue [1 year preoperative]; orange [immediately preoperative]) and the first postoperative scan (D) (blue, immediately postoperative) demonstrated extensive rostrocaudal involvement of the CST with further changes in the body of the corpus callosum (medially coursing fibers). At the last scan 4 months after surgery (D, green), rostral CST on the contralateral side was also seen as being involved. Figure is available in color online only.

of the mass effect, leading to resolution of the associated neurological deficits over time, as demonstrated here. The surgical approach is therefore modified to obviate the risks associated with the resection. Our current approach with respect to the partially disrupted white matter tracts is similar in terms of preventing further injury. Neurological deficits attributable to the disruption, however, may not resolve entirely after the lesionectomy.

\section{Tractography-Based Quantitative Data and Implications}

It is noteworthy that the CST segments in the brainstem as well as in the subcortical region adjacent to their origin are subject to crossing fiber conditions, making QA an appropriate quantitative index for their assessment.

The lower values for mean QA for the affected CST (Table 1) are likely consistent with repeated hemorrhagic episodes in patients with brainstem CMs and the consequent disruption of the tracts. Overall QA may be unable to detect lesser degrees of changes in specific segments of the tract without a craniocaudal compromise. ${ }^{1}$ An example of the latter is the patient in Case 3 with a more rostral (thalamic) CM and multiple hemorrhagic episodes prior to surgery.

To characterize longitudinal changes in the craniocaudal integrity of the CST, we used the specific segment's analysis. The majority of the values (Table 2) represented a compromise of both the perilesional and the nonperile- sional segments. The highest decrease in the QA value for the perilesional segment was unsurprisingly noted for the patient in Case 3 with a history of multiple hemorrhagic episodes over a year prior to the surgical intervention. The patients in Cases 1 and 2, however, underwent resection soon after the initial presentation with symptomatic hemorrhage.

These changes (Table 2 and Supplemental Table) longitudinally evolved predominantly in the perilesional segment and the segment distal to the lesion (Segment 3). Further decreases in the mean QA values in the most caudal segment (Cases 1 and 3) are potentially consistent with wallerian degeneration occurring over months..$^{30,34,45}$ Acute compromise of the fiber tract integrity (e.g., due to hematoma) may be evident using FA within days of the event, ${ }^{49}$ with further changes due to wallerian degeneration being observed within months..$^{30}$ It is noteworthy that the perilesional decreases in the QA values either deteriorated or stayed stable over the follow-up period and after the remov$\mathrm{al}$ of the $\mathrm{CM}$ and associated hemorrhagic products. The susceptibility effects from the paramagnetic molecules, such as deoxyhemoglobin, are known to affect the quantitative results, leading to artifactual signal loss. ${ }^{14}$ Although the susceptibility artifact cannot be completely excluded, the persistence or worsening of the decreases in the perilesional QA values over longitudinal scans points against a major contribution from this phenomenon to our results. 
In a previous study ${ }^{49}$ involving 17 patients with intracerebral hemorrhage ( $\mathrm{ICH}$ ), FA ratios (hematoma side/ contralateral side) were calculated at the level of the hematoma (basal ganglia and/or thalamus) in the CST. DTI was performed within 5 days after the onset of ICH. FA ratio, measured shortly after the $\mathrm{ICH}$, correlated well with the motor functional outcome at 3 months. The study suggested a potential prognostic role for FA in determining patients' eventual motor outcome. FA was not measured in other segments of the CST due to the difficulty of underestimation of FA in areas with multiple crossing fibers. In our small series, despite improvement in their neurological status over time all patients were noted to have some residual deficit at the last follow-up (Table 2).

\section{Diffusion Connectometry Approach-Based Results and Implications}

The results from the connectometry analysis (Fig. 6) were consistent with the quantitative analysis of the specific CST segments. The changes in the rostrocaudal involvement of the CST approximately corresponded to the changes in the QA values for the perilesional and nonperilesional segments. Interestingly in Case 3, the body of the $\mathrm{CC}$ was observed as being affected. These retrograde changes could be potentially attributable to the previously described progressive degenerative transsynaptic effect observed in patients with subcortical stroke involving the pyramidal tract. ${ }^{36}$ Progressive decrease in the FA ratios of the callosal motor fibers correlating with the FA ratios of the pyramidal tract have been demonstrated in this patient group using a DTI study and were thought to be a morphological correlate of transcallosal disinhibition. ${ }^{36} \mathrm{It}$ is unclear whether the rostral changes in Case 3 in the contralateral CST at the last follow-up represent a compensatory phenomenon or a potential weakness of our analytical technique. Further longitudinal data acquisition in a larger series will clarify the relevance of these retrograde changes.

\section{Tractography Versus Diffusion Connectometry Approach: General Limitations and Future Directions}

Both tractography and diffusion connectometry provide complementary information with individual limitations and potentially different applications. A tractography-based approach provides important qualitative data for surgical trajectory planning, as demonstrated here and in our previous studies with supratentorial CMs and tumors. ${ }^{1,15,16}$ The tractography-based quantitative data are derived from comparisons with the contralateral homologous normal tract within the same subject. This could be a potential limitation in the brainstem in the setting of a large mass lesion. Another limitation pertinent to demonstrating the utility of the intrasubject tractography data for surgical planning is the lack of a control group including patients treated without the information from HDFT. A comparison group may potentially better elucidate the utility of HDFT. The obvious difficulty relates to the reported high morbidity associated with the resection of brainstem CMs. ${ }^{4}$

The tractography-based approach is reliant on the user expertise in terms of selection of ROIs and segmentation of the tracts. These, therefore, lead to reproducibility issues, particularly when multiple users are involved. ${ }^{9}, 13,29,39$ To improve this, the key emphasis at our center remains the use of anatomically valid and consistent ROIs. The tractography parameters are defined and kept constant for the specific tracts. Automated diffusion connectometry analysis is a better approach in terms of reproducibility. In the setting of mass lesions, an abnormal trajectory of a fiber may be either technical in origin or real., ${ }^{1,23}$ Despite the improved ability of the advanced techniques ${ }^{22,50}$ to depict white matter tracts in the perilesional edematous zone, a careful interpretation of the qualitative data should be carried out with a sound knowledge of the loco-regional neuroanatomy to facilitate safer surgical planning. Relevant to the quantitative analysis in the longitudinal studies is the variability in the parameters, such as QA, that can occur across the scanning sessions. To ascertain this and the normal range of differences in anisotropy values that can exist between sides (left vs right) related to hemisphere dominance, we are currently scanning healthy subjects on multiple occasions. Establishing these parameters will assist further with interpretation of the quantitative findings in longitudinal studies.

It is noteworthy that the connectometry analysis is not reliant on the contralateral homologous tract. The results are therefore, not completely equivalent to the intrasubject tractography-based analysis. The qualitative data using this approach are also not directly useful for surgical approach planning.

Despite the longitudinal nature of our current study, an obvious limitation is the small sample size. Despite our previous studies involving HDFT and intracranial mass lesions, $, 3,15,16$ the preliminary nature of the findings makes it necessary to exercise caution while interpreting the qualitative and quantitative data for surgical trajectory planning and characterizing changes in the perilesional white matter for neurological prognostication, respectively. ${ }^{1,16} \mathrm{By}$ acquiring more data longitudinally in patients with mass lesions, we are further validating the prognostic potential of a combination of these approaches. The impact of larger lesional pathologies like tumors on the connectometry analysis also needs further evaluation.

The tractography-based data in combination with connectometry data are being evaluated longitudinally for correlation with tract-specific neurological symptoms and eventual clinical outcomes. It will be crucial to identify a threshold for a decrease in the anisotropy values for the perilesional and/or the nonperilesional segment that may correlate with neurological recovery or a permanent deficit. This will move the paradigm beyond the correlation of potential visualization of affected tracts with the eventual neurological outcome.

\section{Conclusions}

Hemorrhagic brainstem CMs can disrupt and displace the perilesional CST, with the latter occurring in unpredictable directions. Tractography-based information can be used to longitudinally monitor the craniocaudal integrity of CST and detect potential wallerian degeneration. Dif- 
fusion connectometry analysis can provide complementary longitudinal information on the extent of rostrocaudal involvement of CST. Although the excellent outcome of these 3 patients cannot be absolutely predicated on the use of this novel technology in this preliminary series, HDFT is clearly an important adjunct for the precise delineation of the critical white matter tracts to optimize the surgical entry point and removes one aspect of uncertainty in the surgical management of such complex and risky lesions.

\section{Acknowledgments}

We would like to acknowledge the Pittsburgh Foundation (Copeland Fund), the Fine Foundation, and the University of Pittsburgh Brain Institute for their support with this study.

\section{References}

1. Abhinav K, Pathak S, Richardson RM, Engh J, Gardner P, Yeh FC, et al: Application of high-definition fiber tractography in the management of supratentorial cavernous malformations: a combined qualitative and quantitative approach. Neurosurgery 74:668-681, 2014

2. Abhinav K, Yeh FC, El-Dokla A, Ferrando LM, Chang YF, Lacomis D, et al: Use of diffusion spectrum imaging in preliminary longitudinal evaluation of amyotrophic lateral sclerosis: development of an imaging biomarker. Front Hum Neurosci 8:270, 2014

3. Abhinav K, Yeh FC, Pathak S, Suski V, Lacomis D, Friedlander RM, et al: Advanced diffusion MRI fiber tracking in neurosurgical and neurodegenerative disorders and neuroanatomical studies: a review. Biochim Biophys Acta 1842:2286-2297, 2014

4. Abla AA, Lekovic GP, Turner JD, de Oliveira JG, Porter R, Spetzler RF: Advances in the treatment and outcome of brainstem cavernous malformation surgery: a single-center case series of 300 surgically treated patients. Neurosurgery 68:403-415, 2011

5. Abla AA, Spetzler RF: Brainstem cavernoma surgery: the state of the art. World Neurosurg 80:44-46, 2013

6. Alexander AL, Hasan KM, Lazar M, Tsuruda JS, Parker DL: Analysis of partial volume effects in diffusion-tensor MRI. Magn Reson Med 45:770-780, 2001

7. Alexander DC, Barker GJ: Optimal imaging parameters for fiber-orientation estimation in diffusion MRI. Neuroimage 27:357-367, 2005

8. Barrow DL, Schuette AJ: Cavernous malformations: a paradigm for progress. Clin Neurosurg 58:27-41, 2011

9. Besseling RM, Jansen JF, Overvliet GM, Vaessen MJ, Braakman HM, Hofman PA, et al: Tract specific reproducibility of tractography based morphology and diffusion metrics. PLoS ONE 7:e34125, 2012

10. Chen L, Zhao Y, Zhou L, Zhu W, Pan Z, Mao Y: Surgical strategies in treating brainstem cavernous malformations. Neurosurgery 68:609-621, 2011

11. Chen X, Weigel D, Ganslandt O, Buchfelder M, Nimsky C: Diffusion tensor imaging and white matter tractography in patients with brainstem lesions. Acta Neurochir (Wien) 149:1117-1131, 2007

12. Chen X, Weigel D, Ganslandt O, Fahlbusch R, Buchfelder M, Nimsky C: Diffusion tensor-based fiber tracking and intraoperative neuronavigation for the resection of a brainstem cavernous angioma. Surg Neurol 68:285-291, 2007

13. Chung HW, Chou MC, Chen CY: Principles and limitations of computational algorithms in clinical diffusion tensor MR tractography. AJNR Am J Neuroradiol 32:3-13, 2010

14. Dorenbeck U, Schlaier J, Bretschneider T, Schuierer G, Feuerbach S: Diffusion-weighted imaging with calculated apparent diffusion coefficient in intracranial hemorrhagic lesions. Clin Imaging 29:86-93, 2005

15. Fernandez-Miranda JC, Engh JA, Pathak SK, Madhok R, Boada FE, Schneider W, et al: High-definition fiber tracking guidance for intraparenchymal endoscopic port surgery. J Neurosurg 113:990-999, 2010

16. Fernandez-Miranda JC, Pathak S, Engh J, Jarbo K, Verstynen T, Yeh FC, et al: High-definition fiber tractography of the human brain: neuroanatomical validation and neurosurgical applications. Neurosurgery 71:430-453, 2012

17. Fernández-Miranda JC, Rhoton AL Jr, Alvarez-Linera J, Kakizawa Y, Choi C, de Oliveira EP: Three-dimensional microsurgical and tractographic anatomy of the white matter of the human brain. Neurosurgery 62 (6 Suppl 3):989-1028, 2008

18. Greenberg AS, Verstynen T, Chiu YC, Yantis S, Schneider W, Behrmann M: Visuotopic cortical connectivity underlying attention revealed with white-matter tractography. J Neurosci 32:2773-2782, 2012

19. Jarbo K, Verstynen T, Schneider W: In vivo quantification of global connectivity in the human corpus callosum. Neuroimage 59:1988-1996, 2012

20. Kim DS, Park YG, Choi JU, Chung SS, Lee KC: An analysis of the natural history of cavernous malformations. Surg Neurol 48:9-18, 1997

21. Konovalov A, Samii M, Porter RW, Spetzler RF, Houteville JP, Yoshimoto T, et al: Brainstem cavernoma. Surg Neurol 54:418-421, 2000

22. Kuhnt D, Bauer MH, Sommer J, Merhof D, Nimsky C: Optic radiation fiber tractography in glioma patients based on high angular resolution diffusion imaging with compressed sensing compared with diffusion tensor imaging - initial experience. PLoS ONE 8:e70973, 2013

23. Lazar M, Alexander AL, Thottakara PJ, Badie B, Field AS: White matter reorganization after surgical resection of brain tumors and vascular malformations. AJNR Am J Neuroradiol 27:1258-1271, 2006

24. Le Bihan D, Poupon C, Amadon A, Lethimonnier F: Artifacts and pitfalls in diffusion MRI. J Magn Reson Imaging 24:478-488, 2006

25. Lekovic GP, Gonzalez LF, Khurana VG, Spetzler RF: Intraoperative rupture of brainstem cavernous malformation. Case report. Neurosurg Focus 21(1):e14, 2006

26. Li D, Hao SY, Jia GJ, Wu Z, Zhang LW, Zhang JT: Hemorrhage risks and functional outcomes of untreated brainstem cavernous malformations. J Neurosurg 121:32-41, 2014

27. MacDonald JD, Antonelli P, Day AL: The anterior subtemporal, medial transpetrosal approach to the upper basilar artery and ponto-mesencephalic junction. Neurosurgery 43:84-89, 1998

28. Mai JC, Ramanathan D, Kim LJ, Sekhar LN: Surgical resection of cavernous malformations of the brainstem: evolution of a minimally invasive technique. World Neurosurg 79:691-703, 2013

29. Malykhin N, Concha L, Seres P, Beaulieu C, Coupland NJ: Diffusion tensor imaging tractography and reliability analysis for limbic and paralimbic white matter tracts. Psychiatry Res 164:132-142, 2008

30. McDonald CR, Hagler DJ Jr, Girard HM, Pung C, Ahmadi ME, Holland D, et al: Changes in fiber tract integrity and visual fields after anterior temporal lobectomy. Neurology 75:1631-1638, 2010

31. McLaughlin N, Kelly DF: Corticospinal tractography as a prognosticator for motor improvement after brainstem cavernoma resection. Br J Neurosurg 27:108-110, 2013

32. Monaco EA, Khan AA, Niranjan A, Kano H, Grandhi R, Kondziolka D, et al: Stereotactic radiosurgery for the treatment of symptomatic brainstem cavernous malformations. Neurosurg Focus 29(3):E11, 2010 
33. Oouchi H, Yamada K, Sakai K, Kizu O, Kubota T, Ito H, et al: Diffusion anisotropy measurement of brain white matter is affected by voxel size: underestimation occurs in areas with crossing fibers. AJNR Am J Neuroradiol 28:11021106, 2007

34. Pierpaoli C, Barnett A, Pajevic S, Chen R, Penix LR, Virta A, et al: Water diffusion changes in Wallerian degeneration and their dependence on white matter architecture. Neuroimage 13:1174-1185, 2001

35. Porter RW, Detwiler PW, Spetzler RF, Lawton MT, Baskin JJ, Derksen PT, et al: Cavernous malformations of the brainstem: experience with 100 patients. J Neurosurg 90:50-58, 1999

36. Radlinska BA, Blunk Y, Leppert IR, Minuk J, Pike GB, Thiel A: Changes in callosal motor fiber integrity after subcortical stroke of the pyramidal tract. J Cereb Blood Flow Metab 32:1515-1524, 2012

37. Royo A, Utrilla C, Carceller F: Surgical management of brainstem-expanding lesions: the role of neuroimaging. Semin Ultrasound CT MR 34:153-173, 2013

38. Shah MV, Heros RC: Microsurgical treatment of supratentorial lesions, in Awad IA, Barrow DL (eds): Cavernous Malformations. Park Ridge, IL: American Association of Neurological Surgeons, 1993, pp 101-116

39. Tensaouti F, Lahlou I, Clarisse P, Lotterie JA, Berry I: Quantitative and reproducibility study of four tractography algorithms used in clinical routine. J Magn Reson Imaging 34:165-172, 2011

40. Ulrich NH, Kockro RA, Bellut D, Amaxopoulou C, Bozinov O, Burkhardt JK, et al: Brainstem cavernoma surgery with the support of pre- and postoperative diffusion tensor imaging: initial experiences and clinical course of 23 patients. Neurosurg Rev 37:481-492, 2014

41. Verstynen T, Jarbo K, Pathak S, Schneider W: In vivo mapping of microstructural somatotopies in the human corticospinal pathways. J Neurophysiol 105:336-346, 2011

42. Verstynen TD, Badre D, Jarbo K, Schneider W: Microstructural organizational patterns in the human corticostriatal system. J Neurophysiol 107:2984-2995, 2012

43. Wang Y, Fernández-Miranda JC, Verstynen T, Pathak S, Schneider W, Yeh FC: Rethinking the role of the middle longitudinal fascicle in language and auditory pathways. Cereb Cortex 23:2347-2356, 2013

44. Wedeen VJ, Hagmann P, Tseng WY, Reese TG, Weisskoff RM: Mapping complex tissue architecture with diffusion spectrum magnetic resonance imaging. Magn Reson Med 54:1377-1386, 2005

45. Wieshmann UC, Symms MR, Clark CA, Lemieux L, Fran- coni F, Parker GJ, et al: Wallerian degeneration in the optic radiation after temporal lobectomy demonstrated in vivo with diffusion tensor imaging. Epilepsia 40:1155-1158, 1999

46. Yeh FC, Tang PF, Tseng WY: Diffusion MRI connectometry automatically reveals affected fiber pathways in individuals with chronic stroke. Neuroimage Clin 2:912-921, 2013

47. Yeh FC, Tseng WY: NTU-90: a high angular resolution brain atlas constructed by q-space diffeomorphic reconstruction. Neuroimage 58:91-99, 2011

48. Yeh FC, Verstynen TD, Wang Y, Fernández-Miranda JC, Tseng WY: Deterministic diffusion fiber tracking improved by quantitative anisotropy. PLoS ONE 8:e80713, 2013

49. Yoshioka H, Horikoshi T, Aoki S, Hori M, Ishigame K, Uchida M, et al: Diffusion tensor tractography predicts motor functional outcome in patients with spontaneous intracerebral hemorrhage. Neurosurgery 62:97-103, 2008

50. Zhang H, Wang Y, Lu T, Qiu B, Tang Y, Ou S, et al: Differences between generalized q-sampling imaging and diffusion tensor imaging in the preoperative visualization of the nerve fiber tracts within peritumoral edema in brain. Neurosurgery 73:1044-1053, 2013

51. Zolal A, Hejčl A, Vachata P, Bartoš R, Humhej I, Malucelli A, et al: The use of diffusion tensor images of the corticospinal tract in intrinsic brain tumor surgery: a comparison with direct subcortical stimulation. Neurosurgery 71:331-340, 2012

\section{Author Contributions}

Conception and design: Friedlander, Faraji, Abhinav, Pathak, Schneider, Fernandez-Miranda. Acquisition of data: Faraji, Abhinav, Hirsch, Schneider. Analysis and interpretation of data: Friedlander, Faraji, Abhinav, Jarbo, Yeh, Shin, Pathak, Schneider, Fernandez-Miranda. Drafting the article: Friedlander, Faraji, Abhinav, Jarbo, Yeh, Shin. Study supervision: Friedlander.

\section{Supplemental Information}

Online-Only Content

Supplemental material is available with the online version of the article.

Supplemental Material. http://thejns.org/doi/suppl/10.3171/ 2014.12.JNS142169.

\section{Correspondence}

Robert M. Friedlander, Department of Neurological Surgery, UPMC Presbyterian, 200 Lothrop St., Ste. B-400, Pittsburgh, PA 15213-2582. email: friedlanderr@upmc.edu. 